\title{
Silicon Electro-optic Modulators Based on p-i-n Diodes Integrated Microdisk and Microring Resonators
}

\author{
Linjie Zhou and Andrew W. Poon \\ Department of Electrical and Electronic Engineering, The Hong Kong University of Science and Technology, \\ Clear Water Bay, Hong Kong SAR, China \\ Tel: (852)-2358-7905; Fax:(852)-2358-1485; Email: eeawpoon@ust.hk
}

\begin{abstract}
We report silicon electro-optic modulators based on p-i-n diodes integrated microdisk and microring resonators. Our experiments demonstrate a 380-MHz 20-micron-size microdisk modulator and a 1-GHz 50-micron-size microring modulator, using $\pm 1 \mathrm{~V}$ driving voltages.

(C) 2006 Optical Society of America

OCIS codes: (230.5750) Resonators; (230.4110) Modulators
\end{abstract}

Recently, silicon electro-optic modulators based on p-i-n diodes integrated microresonators have attained telecommunication-relevant $\mathrm{GHz}$ bandwidth [1]. Although the principle of light intensity modulation through free carrier dispersion induced resonance wavelength shifts has been well recognized, the detailed bandwidth-limiting effects of such p-i-n diodes integrated microresonator-based modulators have yet been thoroughly addressed. Here we study p-i-n diodes integrated silicon microdisk and microring electro-optic modulators in order to distinguish the bandwidth limitations imposed by free carrier transitions and resonance linewidths.
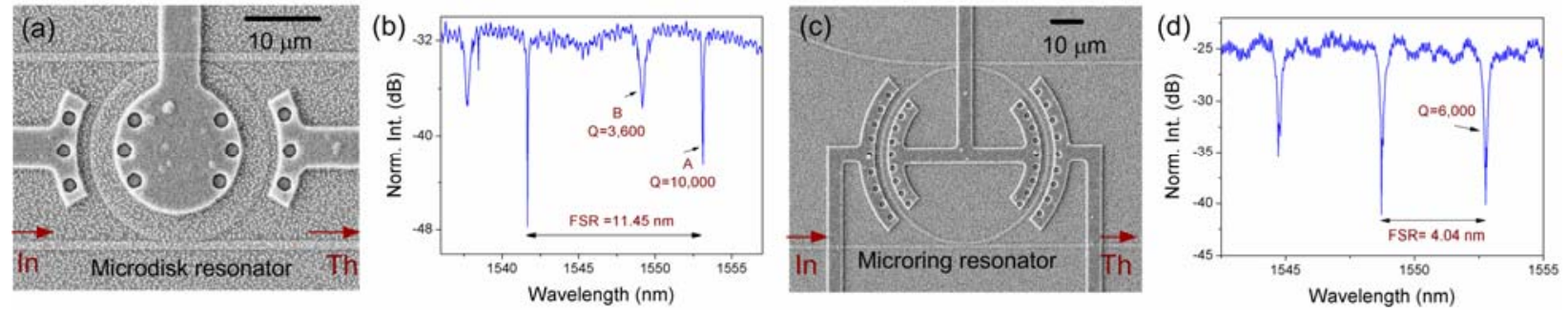

Fig.1. (a) Top-view scanning electron micrograph of a silicon electro-optic modulator based on a p-i-n diodes integrated 20- $\mu \mathrm{m}$-diameter microdisk resonator. (b) Measured multimode throughput spectrum of the microdisk modulator at zero drive voltage. (c) Top-view scanning electron micrograph of a silicon electro-optic modulator based on a p-i-n diodes integrated 50- $\mu \mathrm{m}$-diameter microring resonator. (d) Measured singlemode throughput spectrum of the microring modulator at zero drive voltage. In: input-port, Th: throughput-port.

Figs. 1(a) and (c) show the scanning electron micrographs of our fabricated microdisk and microring resonator-based modulators on thin silicon-on-insulator (SOI) substrates. For the 20- $\mu$ m-diameter microdisk modulator, we selectively embed an $\mathrm{n}^{+}$-doped $\left(1 \times 10^{20} \mathrm{~cm}^{-3}\right)$ disk in the microdisk central region and two $\mathrm{p}^{+}$-doped $\left(2 \times 10^{19} \mathrm{~cm}^{-3}\right)$ arc regions outside the microdisk, leaving the microdisk rim undoped. Thus, the p-i-n diodes span $1 / 3$ of the microdisk circumference with a $4-\mu \mathrm{m}$-wide intrinsic region in the microdisk rim. For the 50 - $\mu \mathrm{m}$-diameter microring modulator, we employ two pairs of $\mathrm{n}^{+}$- and $\mathrm{p}^{+}$-doped arc regions outside the microring inner and outer sidewalls, resulting in p-i-n diodes spanning $1 / 2$ of the microring circumference with 2.4 - $\mu \mathrm{m}$-wide intrinsic region. Figs. 1(b) and (d) show the measured throughput-port spectra of the microdisk and microring resonators. The microdisk resonator exhibits two resonance modes, with the high-Q mode $A$ of $Q \approx 10^{4}$ and low-Q 
mode $\mathrm{B}$ of $\mathrm{Q} \approx 3,600$. The microring resonator displays a singlemode resonance of $\mathrm{Q} \approx 6,000$.

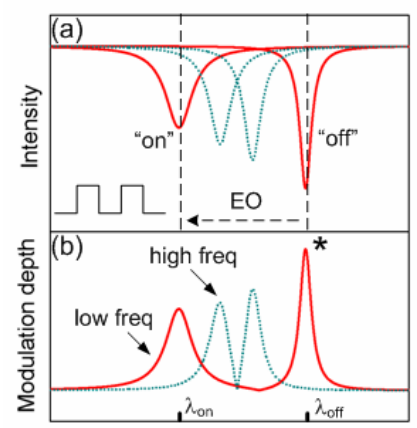

Wavelength
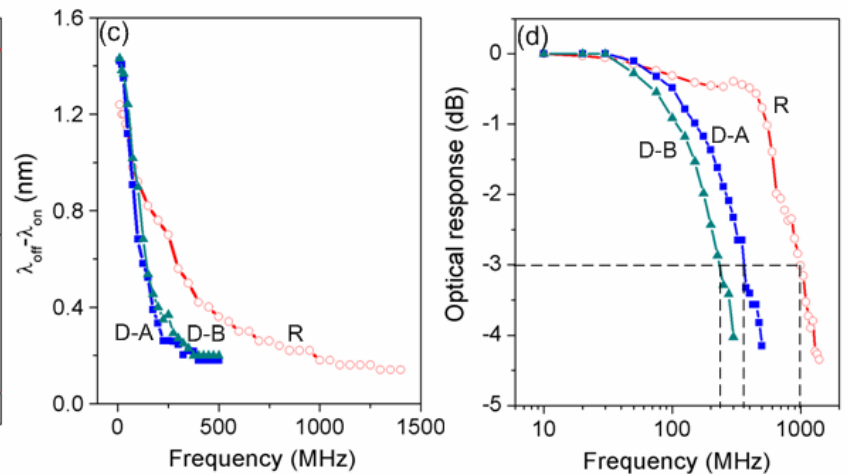

Fig. 2. (a) Schematic of optical resonance modulations under a square-wave electrical signal (inset) of low frequency (solid red line) and high frequency (dotted blue line). EO: electro-optic effect. $\quad$ (b) Schematic of the corresponding optical intensity modulations as a function of probe wavelength. (c) Measured resonance wavelength separation between off- and on-cycles as a function of signal frequency. R: microring (red), D-A: microdisk mode A (blue), D-B: microdisk mode B (green). (d) Measured optical responses (normalized to $10 \mathrm{MHz}$ ) as a function of signal frequency. Dashed lines indicate 3-dB bandwidths.

Fig. 2(a) shows the schematic resonance modulations under a square-wave electrical signal (inset) at a low frequency (solid red line) and a high frequency (dotted blue line). During the on-cycles (forward bias), the resonance is blueshifted and broadened due to the free carrier plasma dispersion effect [2]. During the off-cycles (reverse bias), the resonance is shifted back. Thus, the transmission intensity of a fixed wavelength in the vicinity of on- and off-cycle resonances is modulated. Fig. 2(b) shows the schematic optical intensity modulation depth as a function of wavelength. The resonance wavelength separation $\left(\lambda_{\text {off }}-\lambda_{\text {on }}\right)$ suggests the carrier injection level. At a low signal frequency, carriers can be totally injected and extracted, resulting in a full resonance tuning range. At a high signal frequency, carriers cannot follow the fast drive signal, thus the injected carriers cannot reach saturation during the on-cycles and part of the injected carriers are left as residual carriers during the off-cycles. Thus, both the resonance wavelength tuning range and the corresponding modulation depths reduce with signal frequency. We measure our microdisk and microring modulators optical intensity modulation depths as a function of wavelength at various signal frequencies under $\pm 1 \mathrm{~V}$ biases. Fig. 2(c) shows the measured $\left(\lambda_{\text {off }}-\lambda_{\text {on }}\right)$ as a function of signal frequency. For microdisk modes $\mathrm{A}$ and $\mathrm{B}$, the measured $\left(\lambda_{\text {off }}-\lambda_{\text {on }}\right)$ trends essentially overlap with each other, reflecting modes A and B see the same carrier transitions. However, as mode B has a relatively wide linewidth, the off- and on-cycle resonance lineshapes overlap at a relatively low frequency, resulting in a relatively low bandwidth. Fig. 2(d) shows that mode A exhibits a 3-dB bandwidth of $\sim 380 \mathrm{MHz}$ and mode B displays only $\sim 250 \mathrm{MHz}$ bandwidth. In contrast, the microring mode $\left(\lambda_{\text {off }}-\lambda_{\text {on }}\right)$ reduces considerably slower than the microdisk modes, implying fast carrier transitions in the microring. We attribute this partially to the relatively narrow p-i-n diode intrinsic region, and to the relatively less carriers laterally diffused outside the long p-i-n diode arc regions. Thus, although the microring has a $\mathrm{Q}$ smaller than that of the microdisk mode $\mathrm{A}$, the microring still exhibits a higher bandwidth of $\sim 1 \mathrm{GHz}$ (Fig. 2(d)). Our on-going work focus on enhancing the microdisk modulator bandwidths by using only one coupled waveguide and surrounding the rest of the microdisk rim with p-i-n diodes for less carriers lateral diffusion, and reducing the microdisk size for a potentially narrower intrinsic region.

[1] Q. Xu, B. Schmidt, S. Pradhan, and M. Lipson, “Micrometre-scale silicon electro-optic modulator,” Nature 435, $325-327$ (2005).

[2] R. A. Soref and B. R. Bennett, “Electrooptical effects in silicon,” IEEE J. Quant. Electron 23, 123-129 (1987). 